\title{
The impact of anxiety on conversion from mild cognitive impairment to Alzheimer's disease
}

\author{
Deidre J. Devier ${ }^{1,}{ }^{*}$, Gregory H. Pelton ${ }^{2,3}$, Matthias H. Tabert ${ }^{2,3}$, Xinhua Liu ${ }^{2,3}$, Katrina \\ Cuasay $^{2,3}$, Rachel Eisenstadt 2,3 , Karen Marder 2,3 , Yaakov Stern' 2,3 , and D.P. Devanand 2,3 \\ ${ }^{1}$ Louisiana State University Health Sciences Center, Department of Neurology, New Orleans, LA, \\ USA \\ ${ }^{2}$ Columbia University College of Physicians and Surgeons and New York State Psychiatric Institute, \\ New York, NY, USA \\ ${ }^{3}$ Department of Neurology, Sergievsky Center, Taub Institute, New York, NY, USA
}

\section{SUMMARY}

Objective-To compare state and trait anxiety in mild cognitive impairment (MCI) patients and matched control subjects, and to assess the impact of these variables in predicting conversion to Alzheimer's disease.

Methods-One hundred and forty-eight patients with MCI, broadly defined, were assessed and followed systematically. Baseline predictors for follow-up conversion to AD (entire sample: 39/148 converted to Alzheimer's disease (AD)) included the Spielberger State-Trait Anxiety Inventory (STAI).

Results-At baseline evaluation, MCI patients had higher levels of state and trait anxiety than controls, with no differences between future $\mathrm{AD}$ converters $(n=39)$ and non-converters. In agestratified Cox proportional hazards model analyses, STAI State was not a significant predictor of conversion to $\mathrm{AD}$ (STAI State $\leq 30$ vs. $>30$ risk ratio, $1.68 ; 95 \% \mathrm{CI}, 0.75,3.77 ; p=0.21$ ), but higher Trait scores indicated a lower risk of conversion when STAI State, education, the Folstein MiniMental State Examination and HAM-D (depression score) were also included in the model (STAI Trait $\leq 30$ vs. $>30$ risk ratio, $0.36 ; 95 \%$ CI, $0.16,0.82 ; p=0.015$ ).

Conclusions-In contrast to two other recent studies that showed anxiety predicted cognitive decline or conversion to $\mathrm{AD}$, in this clinic-based sample, state anxiety was not a significant predictor. However, higher Trait anxiety predicted a lower risk of future conversion to AD. Further research with systematic long-term follow-up in larger samples is needed to clarify the role of state and trait anxiety in predicting $\mathrm{MCI}$ conversion to $\mathrm{AD}$.

\section{Keywords}

mild cognitive impairment; Alzheimer's disease; anxiety; depression

\footnotetext{
Copyright $@ 2009$ John Wiley \& Sons, Ltd.

*Correspondence to: D. J. Devier, PhD, LSUHSC/Neurology, 1542 Tulane Avenue, Room 763, New Orleans, LA 70112, USA. E-mail: ddevie@1suhsc.edu.

CONFLICT OF INTEREST

The authors have no conflicts of interest to disclose relevant to this paper or this NIA funded study.
} 


\section{INTRODUCTION}

Mild cognitive impairment (MCI) signifies a transitional stage between age-related memory decline and Alzheimer's disease (AD). The Petersen criteria for MCI require memory complaints, cognitive impairment (test scores $>1.5 \mathrm{SD}$ below age-adjusted norms), and intact daily functioning (Petersen, 2004; Petersen et al., 1999). Conversion rates from MCI to AD, over varying follow-up lengths, range from 4 to $23 \%$ in community-based samples and 10 $31 \%$ in clinic-based samples (Bruscoli and Lovestone, 2004; Luis et al., 2003). This variability is likely related to differences in diagnostic criteria, type of samples, and demographic confounds including age and education.

Patients with MCI or cognitive complaints commonly have comorbid anxiety and depression with rates reported as high as 45 and 65\%, respectively (Palmer et al., 2007; Sinoff and Werner, 2003; Dierckx et al., 2007; Gabryelewicz et al., 2007; Robert et al., 2006; Teng et al., 2007). Comorbid anxiety and depression symptoms can lead to poorer performance on cognitive tests in young and older adults, thus confounding the assessment for MCI (Austin et al., 2001; Coles and Heimberg, 2002; Stroobant and Vingerhoets, 2008; Zakzanis et al., 1998).

The comorbid occurrence of neuropsychiatric symptoms, including anxiety, and memory impairment was associated with continued cognitive decline in community-dwelling elders without cognitive impairment (Sinoff et al., 2003). Another study in a sample of $47 \mathrm{MCI}$ patients found that the presence of anxiety increased the risk of MCI conversion to $\mathrm{AD}$, and endorsement of each additional symptom of anxiety doubled the risk of conversion (risk ratio, 1.8; 95\% CI, 1.2, 2.7; Palmer et al., 2007). Depression, which has been studied more extensively than anxiety in elderly patients with cognitive impairment, is also known to be associated with an increased risk of dementia, and may be a prodromal sign rather than an independent risk factor (Bassuk et al., 1998; Devanand et al., 1996; Wilson et al., 2002).

Clarifying the impact of anxiety on the presentation of MCI, and the effects of these symptoms on cognitive decline and likely conversion to dementia, is important to improve diagnosis and accuracy in estimation of prognosis. In a single-site, long-term study of MCI outpatients without dementia and without an identified cause for cognitive impairment, baseline anxiety measures were compared in patients and healthy control subjects. Further, the impact of baseline state and trait anxiety on the likelihood of conversion to dementia, primarily $\mathrm{AD}$, was examined within this MCI patient sample. Depression scores were also examined with the caveat that since current major depression was an exclusion criterion, the depression scores were low across the sample.

\section{METHODS}

\section{Participants}

Patients presented with memory complaints to either the Memory Disorders Clinic jointly run by the departments of Psychiatry and Neurology at New York State Psychiatric Institute/ Columbia University or the Center for Memory and Behavioral Disorders in the Department of Neurology/Columbia University. All patients met study criteria for cognitive impairment without dementia and without a specific identifiable cause. Healthy control subjects were recruited primarily by advertisement and were required to have cognitive performance within the normal range, and were group-matched to patients on age and sex.

All subjects signed informed consent in this New York State Psychiatric Institute/ Columbia University IRB-approved protocol. 


\section{Eligibility criteria}

The inclusion criteria for patients were intended to define a broad group of cognitively impaired patients with memory complaints and without dementia. Inclusion criteria for patients were age 41-85 years, cognitive impairment between 6 months and 10 years, and Folstein MMSE score $\geq 22 / 30$ (Folstein et al., 1975). Neuropsychological screening inclusion guidelines were Folstein MMSE recall $\leq 2 / 3$ objects at 5 min, or Buschke Selective Reminding Test (SRT) delayed recall score $>1 \mathrm{SD}$ below norms, or Wechsler Adult Intelligence Scale-Revised (WAIS-R) performance IQ score $\geq 10$ points below WAIS-R verbal IQ score. Patients without any of these neuropsychological deficits were considered if they met all of the following three criteria: subjective complaint of memory decline, informant's confirmation of memory decline, and modified Blessed Functional Activity Scale score $\geq 1$ on the first eight items that cover memory-related cognitive and functional problems (Stern et al., 1990). Final determination for inclusion was based on a consensus diagnosis between two expert raters (D.P.D. and Y.S.) who reviewed baseline cognitive, functional and clinical information, neuropsychological test performance, laboratory test results, and MRI clinical report, while remaining blind to all other data.

This study began before MCI criteria were published (Petersen et al., 1999). Since all patients had subjective memory complaints (diagnostic criterion for MCI) and completed an extensive neuropsychological test battery, the baseline MCI subtype could be determined post hoc by using age, education, and sex-based regression norms derived from 83 healthy control subjects (63 from this study and 20 from other studies) who received the same test battery, as previously described (Tabert et al., 2006). Based on this approach, 73\% of patients met criteria for amnestic MCI with or without other cognitive domain deficits, $13.5 \%$ had non-amnestic MCI, and $13.5 \%$ had cognitive scores $<1.5 \mathrm{SD}$ below norms, that is, insufficient to meet MCI criteria (Tabert et al., 2006).

Exclusion criteria included a diagnosis of dementia, schizophrenia, current major affective disorder, active alcohol/substance dependence, clinical history of stroke, cortical stroke or infarct $\geq 2 \mathrm{~cm}$ in diameter based on MRI, cognitive impairment secondary to medications, or other major neurological illness, for example, Parkinson's disease.

For this prospective study, controls were group matched to patients on baseline age and sex. Inclusion criteria for controls were the absence of memory problems, a score of $\geq 34 / 41$ on the Telephone Interview for Cognitive Status, a score of $\geq 27 / 30$ on the MMSE with recall of $\geq 2 / 3$ objects at $5 \mathrm{~min}$, and neuropsychological test scores (using the same battery of tests used to evaluate the patient cohort) that were not more than 1 SD below age adjusted norms. Medical, neurological, and psychiatric exclusion criteria were the same as for patients.

\section{Procedures}

At baseline, the study neurologist/psychiatrist completed history, physical, neurological (including the Unified Parkinson's Disease Rating Scale), and psychiatric examination. Laboratory tests included complete blood count, serum electrolytes, thyroid, liver and renal function tests, rapid plasma reagin (RPR), serum vitamin $B_{12}$ and folate levels, and MRI brain scan.

All subjects received a baseline and annual neuropsychological test battery, reviewed by an experienced neuropsychologist (Y.S.). The two expert raters made a consensus diagnosis at each follow-up, while remaining blind to data from prior visits. The diagnoses of dementia were based on Diagnostic and Statistical Manual of Mental Disorders, Fourth Edition (DSMIV) criteria and possible or probable AD on NINCDS-ADRDA criteria (McKhann et al., 
1984). The endpoint of conversion to AD required this diagnosis at any two consecutive annual assessments during the entire follow-up period.

\section{Anxiety and depression evaluation}

State anxiety, though difficult to define in strict temporal terms, is characterized by the presence of tension, worry, nervousness, and apprehension, and is defined as an active emotional state with a certain level of intensity. Trait anxiety is related more to personality type and remains relatively stable over time though it can be influenced by negative evaluations by parents, teachers and peers during childhood (Purdue and Spielberger, 1966) and has been linked with the personality trait of neuroticism (Smoller and Tsuang, 1998). State and Trait anxiety interact so that individuals with high levels of trait anxiety perceive stimuli as more threatening and respond with higher levels of state anxiety (Spielberger et al., 1983). The Spielberger StateTrait Anxiety Inventory (STAI) was administered at baseline (Spielberger et al., 1983). The STAI is a well-established and validated self-report measure with a subset of items for state anxiety and another subset for trait anxiety. The subject answers 20 questions in each subtest using a scale from 1 (not at all) to 4 (very much so). Scores may range from 20 to 80 for each of the two subtests with higher scores indicating higher levels of anxiety. The STAI data were not normally distributed so each subtest was converted into a dichotomous variable of $\leq 30$ (no to low anxiety) or $>30$ (moderate to high anxiety) based on the cutoff previously reported (Glozman, 2004). Since the overall levels of anxiety were low in this MCI sample, this method was used to differentiate between higher and lower levels of anxiety rather than other cut-point methods developed to distinguish between patients with DSM-IV anxiety disorders and those not meeting criteria for diagnosis (Dennis et al., 2007).

Current major depression was an exclusion criterion for the study. We assessed symptoms of depression with the 17-item Hamilton Rating Scale for Depression (HAM-D) (Hamilton, 1960; Hamilton, 1967). The HAM-D was administered as a structured interview by trained raters (inter-rater reliability for total score 0.94 ).

\section{Neuropsychological evaluation}

The neuropsychological test battery included measures of learning and memory, orientation, abstract reasoning, language, attention, and visuospatial ability, as described elsewhere (Tabert et al., 2006). Patients and controls were tested annually on the full neuropsychological assessment battery.

\section{Statistical analyses}

One-way analysis of variance (ANOVA) and chisquare $\left(\chi^{2}\right)$ tests were used to compare continuous and categorical baseline measures of MCI patients to controls, and to compare MCI patients who converted to AD on follow-up evaluation with MCI patients who did not (hereafter referred to as MCI converters and MCI non-converters, respectively).

In Cox Proportional Hazards Regression survival analysis conducted in the entire patient sample ( $n=148$ ) for the entire follow-up period (1-9 years), the outcome variable was the time from baseline to first time-point of AD diagnosis, with censoring by the time-point of last available evaluation. The follow-up duration varied across subjects and the hazard of AD conversion increased with age: in the < 60 age group, 5.7\% (2/35) patients converted to AD; between the ages of 60 and 67, 15.4\% (6/39); from ages 68 to 74, 30.6\% (11/36); and for ages $\geq 75,52.6 \%$ (20/38) converted. Consequently, age-stratified Cox discrete time-regression models were used to examine the effect of the STAI State and Trait and HAM-D on conversion to $\mathrm{AD}$ controlling for age, first individually and then adjusting for other variables. The final Cox models included the following independent variables: STAI State and Trait, education, baseline cognitive performance (MMSE), and HAM-D. 
Analyses were conducted in SPSS Version 11.0 for Mac OS X.

\section{RESULTS}

The mean age of the study sample (148 patients with MCI and 63 controls) was $66.6 \pm 9.7$ years, $55 \%$ were female, and the mean level of education was $15.5 \pm 3.9$ years. The control and patient groups did not differ in age or sex distribution. The patients had significantly fewer years of education and lower MMSE compared to controls. The duration of follow-up was shorter in patients ( $46.6 \pm 24.6$ months) than controls (52.2 \pm 28.4 months) because MCI converters exited the protocol after 2 consecutive annual diagnoses of AD.

Baseline STAI scores within the entire sample (patients + controls) were not significantly correlated with gender, age, or years of education. Higher STAI State and trait scores showed significant correlation with poorer performance on the MMSE $(r=-0.17, p=0.011$ and $r=$ $-0.18, p=0.007$, respectively) and higher scores on the HAM-D $(r=0.54, p<0.001$ and $r=$ $0.58, p<0.001$, respectively). The two subject groups were then analyzed separately. The STAI State and Trait correlated significantly with HAM-D in the patient group $(r=0.52, p<0.001$ and $r=0.56, p<0.001$, respectively) and in the control group ( $r=0.26, p=0.043$ and $r=0.24$, $p=0.046$, respectively). The mean STAI State and Trait and Ham-D scores were significantly higher for patients than for controls (Table 1) with 33\% and 32\% of controls above the cutoff of 30 for State and Trait, respectively, and $61 \%$ and $67 \%$ of patients above the cutoff for the same variables.

Within 6 months of presentation, 2 patients with MCI were clinically diagnosed as having other neurologic disorders (corticobasal degeneration and amyotrophic lateral sclerosis presenting with frontal lobe deficits), and were excluded from analyses. Thirty-nine (26.4\%) of the remaining 148 patients enrolled in the longitudinal study converted to AD during the entire follow-up period. All converters were $>55$ years old at baseline evaluation. Of the 39 converters, 31 had probable $\mathrm{AD}$ and 8 had possible $\mathrm{AD}$, that is, $\mathrm{AD}$ with concomitant conditions that developed during follow-up (depression $n=6$, prescription drug abuse $n=1$, Parkinsonian features without meeting criteria for Lewy Body Dementia $n=1$ ). The clinical course and decline in cognitive scores during follow-up did not differ between the probable and possible $\mathrm{AD}$ converters who were combined for data analysis.

MCI converters and non-converters did not differ in sex distribution, education, or baseline HAM-D scores, but converters were older and scored lower on the MMSE at baseline (Table 1). MCI converters did not differ from non-converters on mean STAI State or Trait scores.

In an age-stratified Cox Model, HAM-D was not a significant predictor alone or with education and MMSE also included in the model (risk ratio, $0.993 ; 95 \% \mathrm{CI}, 0.92,0.072 ; p=0.862$ ). The dichotomous predictors $(\leq 30$ or $>30$ ) STAI State and Trait, were entered into age-stratified Cox proportional hazards models, first as single predictors and then with education, MMSE, and HAM-D in the model (Table 2). STAI State was not significant as a single predictor of conversion to AD or after controlling for the other variables. STAI Trait did not predict conversion to $\mathrm{AD}$ as a single predictor (STAI Trait $\leq 30 \mathrm{vs}$. $>30$ risk ratio, $0.61 ; 95 \% \mathrm{CI}, 0.32$, $1.17 ; p=0.13$ ), while higher STAI Trait scores (>30) were significantly associated with a lower risk of developing $\mathrm{AD}$ (risk ratio, $0.45 ; 95 \% \mathrm{CI}, 0.21,0.95 ; p=0.036$ ) when education and MMSE were include in the model, when HAM-D was and was not in the model, and remained significant when STAI State was also added into the model (Table 2). The same analyses were conducted in only the 108 amnestic MCI patients who met criteria for "pure" amnestic MCI $(n=21)$ or amnestic MCI with deficits in multiple cognitive domains $(n=87)$. The results were similar to those obtained in the entire sample of 148 patients. Higher STAI Trait anxiety scores were associated with a lower risk of conversion from amnestic MCI to AD 
(STAI Trait $\leq 30$ vs. $>30$ risk ratio, $0.35 ; 95 \% \mathrm{CI}, 0.14,0.85 ; p=0.02$ ) when STAI State, education, MMSE, and HAM-D were included in the model (Figure 1).

We also used a Cox Model with age classified as a dichotomous variable ( $<75$ or $\geq 75$ ), because $20 / 39$ (52\%) of the converters were $\geq 75$ and in order to make the sample comparable to the Palmer et al. (2007) study. In the model including STAI State and Trait, education, MMSE and HAM-D, State anxiety was not a significant predictor (STAI State $\leq 30$ vs. $>30$ risk ratio, $1.55 ; 95 \%$ CI, $0.72,3.37 ; p=0.26$ ) while Trait anxiety remained significant (STAI Trait $\leq 30$ vs. $>30$ risk ratio, $0.41 ; 95 \% \mathrm{CI}, 0.19,0.91 ; p=0.028)$. Trait remained significant when STAI State was also included in the model.

\section{DISCUSSION}

In this clinic-based sample, the MCI patients had higher levels of both state and trait anxiety compared to controls, consistent with the reported literature regarding the increased prevalence of neuropsychiatric symptoms in persons with MCI or complaints of memory impairment (Feldman et al., 2004; Forsell et al., 2003; Palmer et al., 2007; Sinoff and Werner, 2003). Our study enrolled a relatively large cohort of well-characterized patients with MCI and a smaller group of normal control participants. One strength of the study design was the measurement of both state and trait anxiety and depression. The STAI has been used extensively across numerous research studies. There is evidence to support the use of the STAI, which has shown good psychometric properties in elderly samples (Kvaal et al., 2005; Stanley et al., 2001), and has been validated as an outcome variable in geriatric samples (Giraudet-Le Quintrec et al., 2003; Prudente et al., 2006; Wu et al., 2004). The strong correlations between the STAI and HAM-D scores support the view that anxiety and depression are frequently comorbid in elderly patients with cognitive impairment, though our study excluded anyone with major depression, limiting the evaluation of the prevalence of comorbid anxiety and depression in our sample.

In survival analyses, depression scores did not predict conversion, though the entry criterion, that is, exclusion of current major depression, restricted the range in depression scores and hence the interpretation of this finding. STAI State anxiety did not predict conversion during follow-up. This result stands in contrast to other published data indicating that presence of elevated baseline anxiety predicts future cognitive decline in elderly normals (Sinoff and Werner, 2003) or conversion to AD in MCI patients (Palmer et al., 2007).

The HaGenfen study (Sinoff and Werner, 2003) enrolled elderly participants who were cognitively intact at baseline and who were classified as having anxiety or not based on the Sinoff's Short Anxiety Screening Test, an instrument specifically designed to assess anxiety in the elderly (Table 3 ). The anxiety group had significantly more subjective memory complaints at baseline and was more likely to decline cognitively over the follow-up period. Our study measured conversion from MCI to AD, while the HaGenfen study defined cognitive decline based on a follow-up MMSE score <24 (Sinoff and Werner, 2003). For more direct comparison, we ran the same analyses using MMSE $<24$ as an outcome marker for cognitive decline in our patients with baseline MCI and found that State anxiety still did not predict cognitive decline at follow-up (data not shown).

Other methodological and sample differences, discussed below, may account for the discrepancy in results between our study and the Kungsholmen Project (Palmer et al., 2007), which found markedly increased risk of conversion to $\mathrm{AD}$ in MCI patients with anxiety compared to MCI patients without anxiety. The Kungsholmen Project also found in MCI patients (single or multiple cognitive domain deficits) with anxiety, that there was a 30-fold increased risk of conversion to AD compared to cognitively intact controls. They measured anxiety by extracting items from a larger neuropsychiatric inventory, and included state anxiety 
symptoms of indecision, persistent worrying, anxiety, and social withdrawal. The difference in instruments used in the Kungsholmen Project and our study, which limited measurement of anxiety to the STAI, may partly account for the discrepant findings obtained. Additionally, the participants in the Kungsholmen Project demonstrated greater cognitive impairment (MMSE $\leq 24$ ) and were older $(\geq 75)$ at baseline than our sample (Table 3). However, in an analysis of our participants aged 75-plus, State anxiety remained non-significant in predicting conversion to $\mathrm{AD}$. The results did not change when we restricted our analysis to patients meeting criteria for either amnestic MCI or amnestic MCI with multiple cognitive domain deficits.

In our study, in survival analysis, higher levels of trait anxiety predicted a reduced risk of developing $\mathrm{AD}$ when education, MMSE and depression were included in the model. Trait anxiety is relatively stable over time and leads one to view stressful situations as more or less threatening, depending on the trait level, and to respond accordingly (Spielberger et al., 1983). When faced with perceived memory decline, a person with high trait anxiety may be more likely to seek evaluation of memory complaints. These patients may not, in fact, have objective evidence of memory deficits and may be less likely to convert to AD.

Although two other studies suggest that the effect of state anxiety is strong for predicting decline in cognitively normal elders or MCI conversion to $\mathrm{AD}$, our state anxiety results do not support these findings, and the trait anxiety results are somewhat contradictory in that increased trait anxiety was related to a decreased risk of developing AD. Prior studies primarily measured state anxiety and not trait anxiety in MCI. Given the relatively small sample size of MCI subjects in all the studies to date, including our own, more research is clearly needed to clarify the role of anxiety in cognitive decline and conversion to $\mathrm{AD}$, if the strength of the relationship is present, and the underlying mechanisms.

\section{KEY POINTS}

- Higher Trait anxiety is related to a lower risk of conversion from MCI to AD.

- State anxiety is not related to conversion from MCI to AD.

\section{Acknowledgments}

This study was supported in part by grants R01AG17761, R01AG12101, 1K01AG21548, P50 AG08702, and P30 AG08051 from the National Institute on Aging and grants MH55735, MH35636, and MH55646 from the National Institute of Mental Health.

\section{REFERENCES}

Austin MP, Mitchell P, Goodwin GM. Cognitive deficits in depression: possible implications for functional neuropathology. Br J Psychiatry 2001;178:200-206. [PubMed: 11230029]

Bassuk SS, Berkman LF, Wypij D. Depressive symptomatology and incident cognitive decline in an elderly community sample. Arch Gen Psychiatry 1998;55(12):1073-1081. [PubMed: 9862549]

Bruscoli M, Lovestone S. Is MCI really just early dementia? A systematic review of conversion studies. Int Psychogeriatr 2004;16(2):129-140. [PubMed: 15318760]

Coles ME, Heimberg RG. Memory biases in the anxiety disorders: current status. Clin Psychol Rev 2002;22(4):587-627. [PubMed: 12094512]

Dennis RE, Boddington SJ, Funnell NJ. Self-report measures of anxiety: are they suitable for older adults? Aging Ment Health 2007;11(6):668-677. [PubMed: 18074254]

Devanand DP, Sano M, Tang MX, et al. Depressed mood and the incidence of Alzheimer's disease in the elderly living in the community. Arch Gen Psychiatry 1996;53(2):175-182. [PubMed: 8629893]

Dierckx E, Engelborghs S, De Raedt R, et al. Mild cognitive impairment: what's in a name? Gerontology 2007;53(1):28-35. [PubMed: 16988509] 
Feldman H, Scheltens P, Scarpini E, et al. Behavioral symptoms in mild cognitive impairment. Neurology 2004;62(7):1199-1201. [PubMed: 15079026]

Folstein MF, Folstein SE, McHugh PR. Mini-mental state. A practical method for grading the cognitive state of patients for the clinician. J Psychiatr Res 1975;12(3):189-198. [PubMed: 1202204]

Forsell Y, Palmer K, Fratiglioni L. Psychiatric symptoms/syndromes in elderly persons with mild cognitive impairment. Data from a cross-sectional study. Acta Neurol Scand Suppl 2003;179:25-28. [PubMed: 12603247]

Gabryelewicz T, Styczynska M, Luczywek E, et al. The rate of conversion of mild cognitive impairment to dementia: predictive role of depression. Int J Geriatr Psychiatry 2007;22(6):563-567. [PubMed: 17136705]

Giraudet-Le Quintrec JS, Coste J, Vastel L, et al. Positive effect of patient education for hip surgery: a randomized trial. Clin Orthop Relat Res 2003;414:112-120. [PubMed: 12966284]

Glozman, J. Communication Disorders and Personality. Netherland: Springer; 2004.

Hamilton M. A rating scale for depression. J Neurol Neurosurg Psychiatry 1960;23:56-62. [PubMed: 14399272]

Hamilton M. Development of a rating scale for primary depressive illness. Br J Soc Clin Psychol 1967;6 (4):278-296. [PubMed: 6080235]

Kvaal K, Ulstein I, Nordhus IH, et al. The Spielberger State-Trait Anxiety Inventory (STAI): the state scale in detecting mental disorders in geriatric patients. Int J Geriatr Psychiatry 2005;20(7):629-634. [PubMed: 16021666]

Luis CA, Loewenstein DA, Acevedo A, et al. Mild cognitive impairment: directions for future research. Neurology 2003;61(4):438-444. [PubMed: 12939414]

McKhann G, Drachman D, Folstein M, et al. Clinical diagnosis of Alzheimer's disease: report of the NINCDS-ADRDA Work Group under the auspices of Department of Health and Human Services Task Force on Alzheimer's Disease. Neurology 1984;34(7):939-944. [PubMed: 6610841]

Palmer K, Berger AK, Monastero R, et al. Predictors of progression from mild cognitive impairment to Alzheimer disease. Neurology 2007;68(19):1596-1602. [PubMed: 17485646]

Petersen RC. Mild cognitive impairment as a diagnostic entity. J Intern Med 2004;256(3):183-194. [PubMed: 15324362]

Petersen RC, Smith GE, Waring SC, et al. Mild cognitive impairment: clinical characterization and outcome. Arch Neurol 1999;56(3):303-308. [PubMed: 10190820]

Prudente LA, Reigle J, Bourguignon C, et al. Psychological indices and phantom shocks in patients with ICD. J Interv Card Electrophysiol 2006;15(3):185-190. [PubMed: 17019638]

Purdue O, Spielberger C. Anxiety and the perception of punishment. Ment Hyg 1966;50:390.

Robert PH, Berr C, Volteau M, et al. Apathy in patients with mild cognitive impairment and the risk of developing dementia of Alzheimer's disease: a one-year follow-up study. Clin Neurol Neurosurg 2006;108(8):733-736. [PubMed: 16567037]

Sinoff G, Werner P. Anxiety disorder and accompanying subjective memory loss in the elderly as a predictor of future cognitive decline. Int J Geriatr Psychiatry 2003;18(10):951-959. [PubMed: 14533128]

Smoller JW, Tsuang MT. Panic and phobic anxiety: defining phenotypes for genetic studies. Am J Psychiatry 1998;155(9):1152-1162. [PubMed: 9734536]

Spielberger, C.; Gorsuch, R.; Lushene, R., et al. Manual for the state-trait inventory. Palo Alto: Consulting Psychologists Press; 1983.

Stanley MA, Novy DM, Bourland SL, et al. Assessing older adults with generalized anxiety: a replication and extension. Behav Res Ther 2001;39(2):221-235. [PubMed: 11153975]

Stern Y, Hesdorffer D, Sano M, et al. Measurement and prediction of functional capacity in Alzheimer's disease. Neurology 1990;40(1):8-14. [PubMed: 2296387]

Stroobant N, Vingerhoets G. Depression, anxiety, and neuropsychological performance in coronary artery bypass graft patients: a follow-up study. Psychosomatics 2008;49(4):326-331. [PubMed: 18621938]

Tabert MH, Manly JJ, Liu X, et al. Neuropsychological prediction of conversion to Alzheimer disease in patients with mild cognitive impairment. Arch Gen Psychiatry 2006;63(8):916-924. [PubMed: 16894068] 
Teng E, Lu PH, Cummings JL. Neuropsychiatric symptoms are associated with progression from mild cognitive impairment to Alzheimer's disease. Dement Geriatr Cogn Disord 2007;24(4):253-259. [PubMed: 17700021]

Wilson RS, Barnes LL, Mendes de Leon CF, et al. Depressive symptoms, cognitive decline, and risk of AD in older persons. Neurology 2002;59(3):364-370. [PubMed: 12177369]

Wu HS, Wu SC, Lin JG, et al. Effectiveness of acupressure in improving dyspnoea in chronic obstructive pulmonary disease. J Adv Nurs 2004;45(3):252-259. [PubMed: 14720242]

Zakzanis KK, Leach L, Kaplan E. On the nature and pattern of neurocognitive function in major depressive disorder. Neuropsychiatry Neuropsychol Behav Neurol 1998;11(3):111-119. [PubMed: 9742509] 


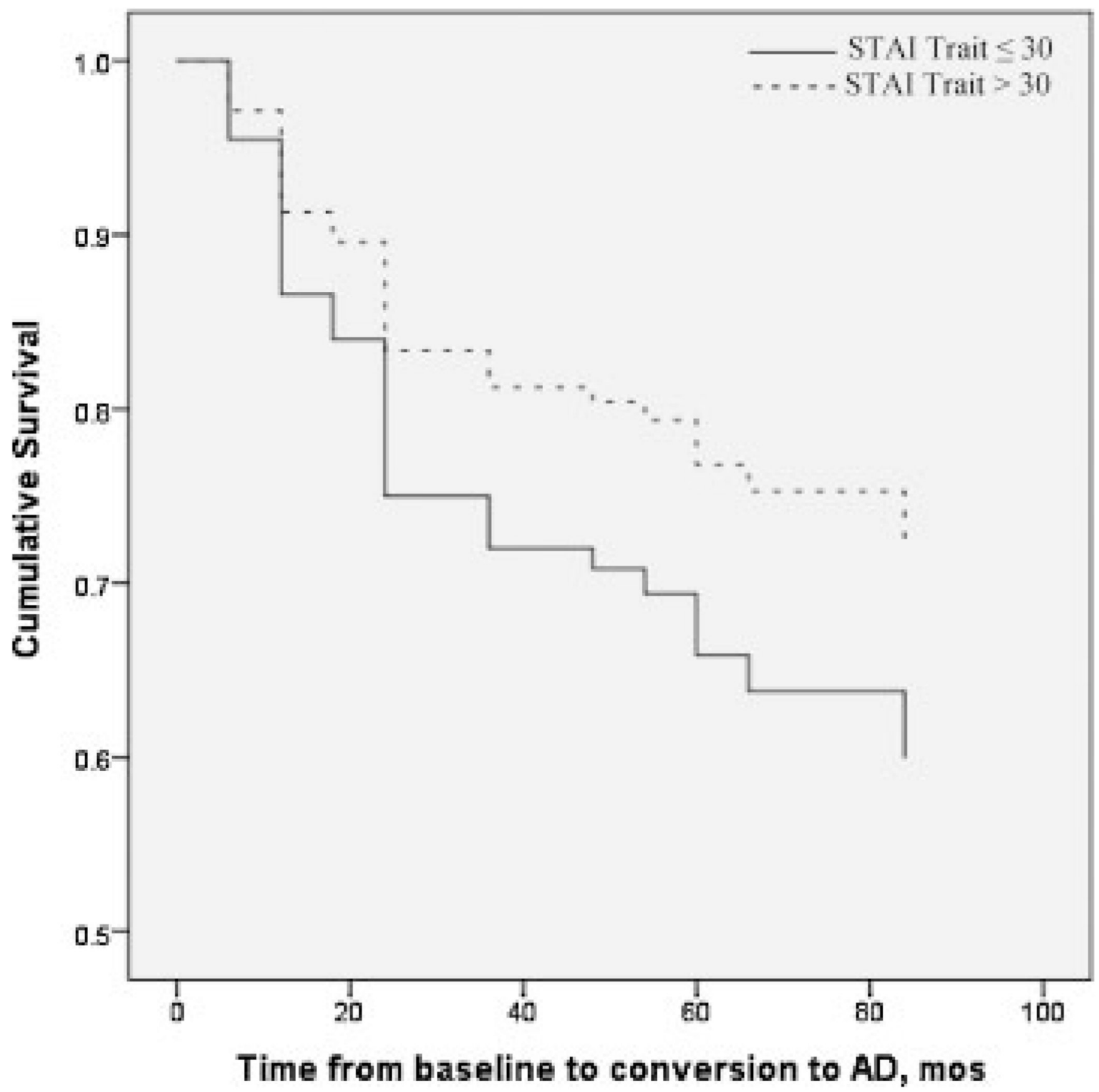

Figure 1.

Effect of Trait Anxiety on Cox Proportional Hazards Model survival time to AD in 148 outpatients with cognitive impairment. Higher Trait anxiety (>30) confers a lower risk of conversion from MCI to $\mathrm{AD}$, higher cumulative survival over time. Cox model also include the variables age, education, MMSE, and HAM-D. 


\section{Table 1}

Descriptive statistics on demographic and clinical variables at baseline by group: MCI patients vs. controls and MCI converters vs. non-converters

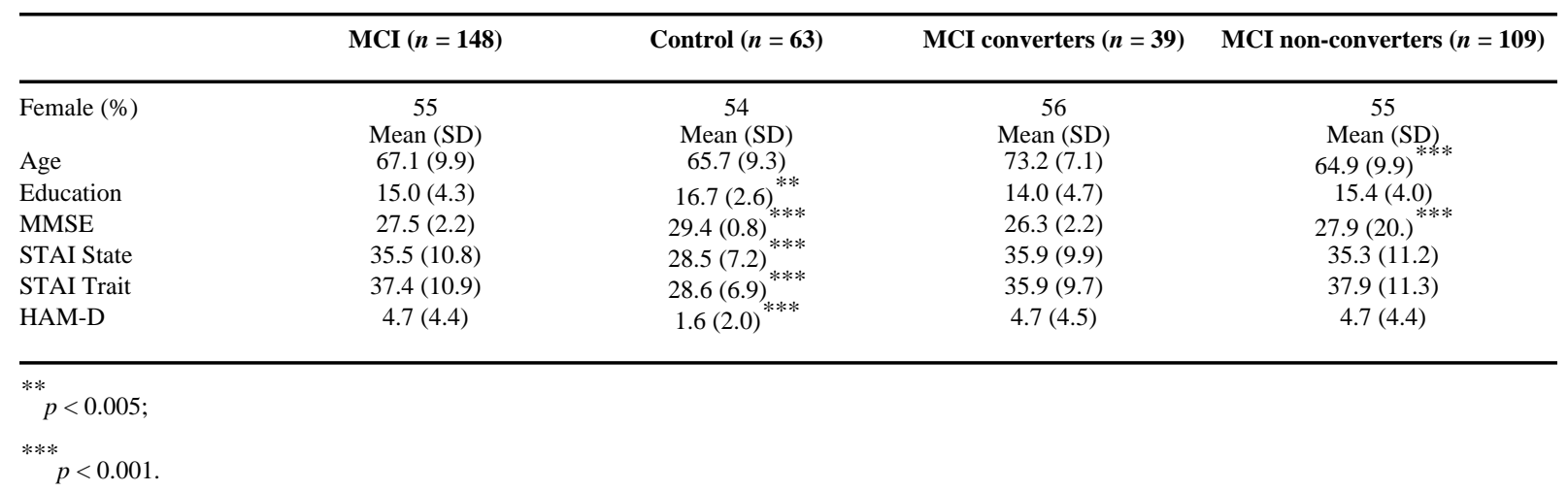


Table 2

Hazards ratios (95\% Confidence Intervals) derived from age-stratified Cox Proportional Hazards models with single predictor of either Spielberger State or Trait anxiety, and with both anxiety variables and control variables of education, baseline MMSE and HAM-D

\begin{tabular}{lcc}
\hline Predictor variables & $\begin{array}{c}\text { Hazards ratio }(\mathbf{9 5 \%} \mathbf{C I}) \\
\text { from single predictor model }\end{array}$ & $\begin{array}{c}\text { Hazards ratio (95\% CI) } \\
\text { adjusted for other variables }\end{array}$ \\
\hline State anxiety $(\leq 30$ vs. $>30)$ & $1.26(0.65,2.44) p=0.49$ & $1.68(0.75,3.77) p=0.21$ \\
Trait anxiety $(\leq 30$ vs. $>30)$ & $0.61(0.32,1.17) p=0.13$ & $0.36(0.16,0.82) p=0.015$ \\
Education & & $1.01(0.93,1.10) p=0.81$ \\
MMSE & & $0.75(0.64,0.87) p<0.001$ \\
HAM-D & $1.01(0.92,1.10) p=0.86$ \\
\hline
\end{tabular}


Table 3

Summary of results from comparative studies

\begin{tabular}{|c|c|c|c|}
\hline & Sinoff and Werner, 2003 & Palmer et al., 2007 & Devier, current study \\
\hline Sample type & Clinic based study & Population based study & Clinic based study \\
\hline Sample size & $\begin{array}{l}42 \text { anxiety, no cognitive } \\
\text { impairment; } 58 \text { controls }\end{array}$ & $\begin{array}{l}47 \text { patients with } \mathrm{MCI} ; 185 \\
\text { controls }\end{array}$ & $\begin{array}{l}148 \text { cognitive impairment ( } 108 \text { amnestic } \\
\text { MCI +/-; } 20 \text { non-amnestic MCI, } 20 \text { no } \\
\text { MCI) } 63 \text { controls }\end{array}$ \\
\hline Age mean (SD) & $\begin{array}{l}\text { Patients: } 75.9(5.0) \\
\text { Controls: } 78.9(6.3)\end{array}$ & Entire sample: $84.0(5.1)$ & $\begin{array}{l}\text { Patients: } 67.1(9.9) \\
\text { Controls: } 65.7(9.3)\end{array}$ \\
\hline Cognitive inclusion & No cognitive impairment & Patients with MCI & Patients with cognitive impairment \\
\hline MMSE & MMSE $\geq 24$ & $\begin{array}{l}\text { Patients: MMSE < } 24 \\
\text { Controls: MMSE > } 24\end{array}$ & $\begin{array}{l}\text { Patients: MMSE } \geq 22 \\
\text { Controls: MMSE } \geq 27\end{array}$ \\
\hline Anxiety scales & $\begin{array}{l}\text { Sinoff's Short Anxiety } \\
\text { Screening Test }\end{array}$ & $\begin{array}{l}\text { Comprehensive Psychopatho- } \\
\text { logical Rating Scale }\end{array}$ & STAI \\
\hline State/Trait & State & State & State and Trait \\
\hline $\begin{array}{l}\text { Average follow-up } \\
\text { duration }\end{array}$ & 3.1 years & 3.4 years & 4.3 years evaluated annually \\
\hline Findings & $\begin{array}{l}\text { Anxiety predicted cognitive } \\
\text { decline }\end{array}$ & $\begin{array}{l}\text { Anxiety predicted conversion } \\
\text { from MCI to AD }\end{array}$ & $\begin{array}{l}\text { State anxiety did not predict conversion from } \\
\text { MCI to AD; Higher Trait anxiety scores } \\
\text { predicted a lower risk of conversion from } \\
\text { MCI to AD }\end{array}$ \\
\hline
\end{tabular}

